\title{
STUDY AND ANALYSIS OF PROTECTION SCHEME OF DIGITAL SUBSTATION USING IEC61850-9-2 PROCESS BUS TECHNOLOGY
}

\author{
Yuvaraj Nayak \\ R V College of Engineering, Bengaluru, India
}

\section{Lakpathi Muniswamy}

Siemens Pvt, Ltd, Bengaluru, India

\section{Mithun T P}

\author{
Assistant Professor Dept of Telecommunication Engineering, \\ R V College of Engineering, Bengaluru, India
}

\begin{abstract}
Substations are a fundamental part in electrical energy transmission and distribution. The role of a substation is to transfer and transform electrical energy by stepping up or down the voltage. To do this, high voltage switching equipment and power transformers are used, in addition to instrument transformers that supply the status of the primary system to the secondary equipment. Substation Automation Systems are then used to control, protect and monitor the substations. The IEC 61850 standard developed digital substation with most advanced techniques. The IEC 61850 standard define in its sub- clauses IEC 600448 and IEC 61850-9-2 about digital interface, digital communication and Sampled Values transmission over an Ethernet link called Process Bus. Process Bus technology mainly developed in order to reduce the usage of copper wiring at substation control by introducing IEC 61850-9-2 digital interface.
\end{abstract}

Keywords: Process Bus, Merging Unit, IEC61850, IEDs, SCADA

Cite this Article: Yuvaraj Nayak, Lakpathi Muniswamy and Mithun T P, Study and Analysis of Protection Scheme of Digital Substation using IEC61850-9-2 Process Bus Technology, International Journal of Electrical Engineering \& Technology, 10(3), 2019, pp. 1-9.

http://iaeme.com/Home/issue/IJEET?Volume=10\&Issue $=3$

\section{INTRODUCTION}

Power System consists of generation, transmission and distribution; safe power system operation is essential to ensure client satisfaction. Power System protection is an essential event for the safety and reliable operation of the power grid. Within substation automation, 
electromechanical relays were the first to start in the protection field. Solid-state relays replaced the first generation of electromechanical relays and were more efficient in the sense of their operation functionalities. Later on the electronics revolution brought microprocessorbased relays -as the state of the art- with many advanced operating principles, the use of which has achieved the highest level of substation automation for more reliable energy output to customers.

In power transmission system, the power substation plays a significant role since one of its roles is to transfer the voltage from high to low or vice versa by utilising the power transformer. In the substation, the monitoring, control, protection and other automation functions are provided by the Substation Automation System (SAS). The communication network within the substation is divided into three levels:

station level, bay level and process level [2]. NCIT and Merging Unit are located on the process level. The communication network between primary equipment on process level and bay level is referred to as Process Bus.

The communication aspect of Process Bus is known as Sampled Values service in the $\mathrm{SAS}$, as per the recommendation of the International Electrotechnical Commission (IEC) standard 61850 for the use of Ethernet based communication in the substation automation field.

At present, active work is under way to develop and introduce digital substations based on modern micro- processing control and relay modules with digital current and voltage instrument (optical) transformers [1, 2, 3]. Leading relay protection manufacturers have already successfully developed and launched production of microprocessor relay protection modules adapted to work with digital flows, using process buses according to Standard 61850-9-2LE. A somewhat more complicated task is the implementation of current and voltage instrument transformers. The simplest form is to use standard current and voltage transformers provided with measuring merging devices (Stand Alone Merging Units). This approach, however, does not fully resolve the problems of standard current and voltage transformers, such as core saturation. Because of their high price, the use of optical transformers is warranted only at voltages exceeding $110-220 \mathrm{kV}$. Using digital (unconventional) transformers opens the possibility of operating them in a wide voltage range, from 6 to $220 \mathrm{kV}$.

The idea of development and implementation of digital substations, based on the application of the IEC 61850 standard, is widely used all over the world. The modern approach to digital substations involves the use of a sufficiently large number of merging units (MU) and intelligent electronic devices (IED), the data transfer between them is carried out through the Local Area Network (LAN) using the SV and GOOSE protocols. While developing the LAN structure it is needed to take into account the amount of traffic produced by SV streams. To ensure reliable operation of protection automation control system (PACS) for digital substation it is necessary to use specialized methods for managing broadcast traffic (VLAN, QoS, etc.). Sophisticated LAN leads to increasing of development and maintenance costs of digital substation.

The use of process-bus technology, designed in accordance with the IEC 61850 standard, introduces new opportunities for protection and control. However, before the technology can be deployed, adequate tests are required to prove use of the process bus does not have a detrimental impact on the operating performance of future protection, as expressed in terms of dependability, security and operating speed. The paper will describe an overview of the concepts and benefits of the process bus, and how it affects the design of prototype protection scheme suitable for the mesh-corner of a transmission substation. The operating characteristics of conventional hardwired relays and IEC 61850-9-2LE relays applied within a 
feeder protection scheme, were compared and assessed against conventional type-test results. The scheme includes Ethernet Switches from different manufacturers, configured to allow the assessment of different process-bus architectures. The effect of interchangeability and time offsets were investigated both experimentally and using the OPNET simulator. The scheme includes Merging Units (MU), tested to assess the magnitude of their time offset and how they drift with time and temperature. Causes of incorrect operation were recognized and recommendations presented to avoid the degradation of system performance. Given an appropriate configuration, the performance of a process bus based protection scheme is comparable to a conventional hardwired scheme. The presented results provides a fundamental reference for all the participating parties to create a roadmap for the efficient application and deployment of protection and control systems based on IEC 61850 process bus and digital Ethernet communications.

The IEC 61850 "Communication Networks and Systems for Utility Automation" standard helps utilities consider new possibilities for secondary systems in new and refurbished substations. The 61850-9-2 sub-set of the standard, referred to as Process-Bus, allows the replacement of conventional analogue and binary signals with Ethernet messages and enables the use of a digital communication link between Merging Units (MUs), the interface to current/voltage transformers and switchgear, and bay devices such as protective relays, bay controllers or meters.

The process-bus will reduce substation costs as it allows the removal of conventional copper wiring and minimizes engineering, commissioning and maintenance work. This, in combination with the free allocation of functions, improves flexibility and functionality.

To ensure utilities accept or embrace the process-bus, it is necessary to prove that the operating performance of protection and control schemes based on 61850-9-2 is equivalent or exceeds that of conventional hardwired schemes. This paper will experimentally assess the feasibility of protecting a feeder using prototype 61850-9-2LE [2] distance and differential relays at the local terminal of the feeder and similar, but conventional hardwired, relays at the remote terminal. Merging Units (MU) and Ethernet Switches from different manufacturers have been integrated into the scheme and issues related to interchangeability and time offsets assessed. The results obtained and the lessons learnt provide a fundamental reference to help utilities gain confidence in the use of process-bus based protection and control schemes.

\section{PROCESS BUS OVERVIEW AND BENEFITS}

The concept of a "digital" substation is based on the use of intelligent primary equipment and networked secondary devices, these share digital information and realize distributed protection and control functions via a common Ethernet network with communication implemented according to 61850 standard. Figure 1 illustrates the conceptual architecture of an IEC 61850 substation. 


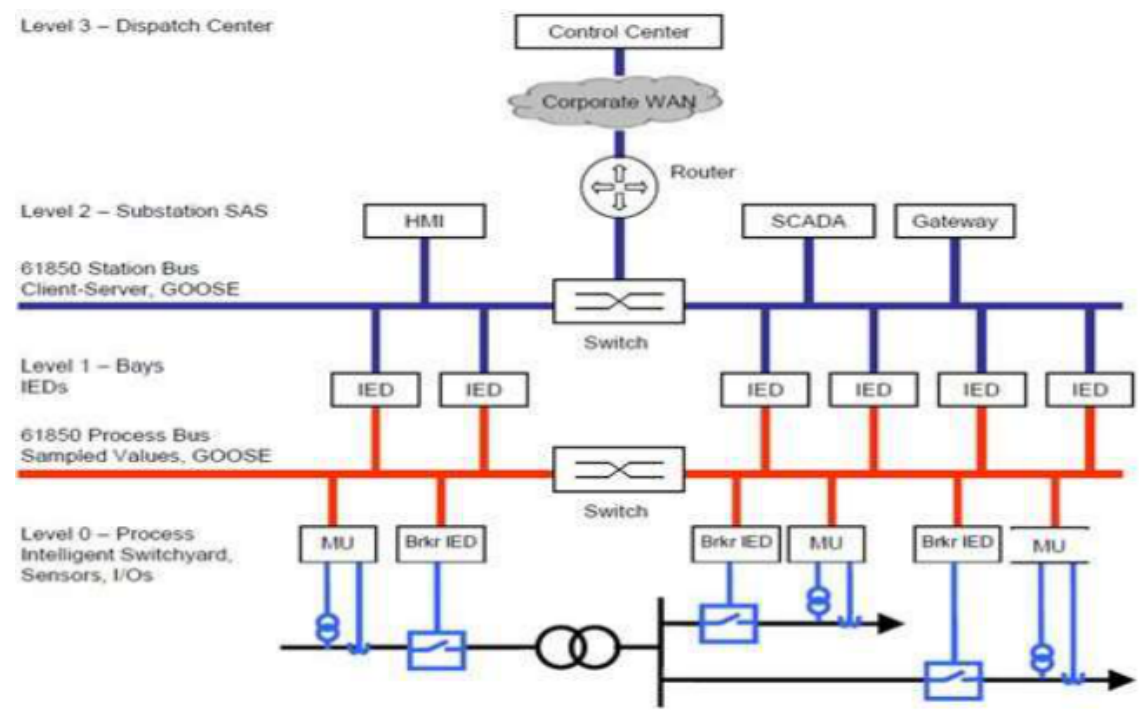

Figure 1 Architecture of IEC 61850 Substation Automation System

In addition to the savings in cabling and wiring, another benefit of using a process bus is it's affect on the retrofitting of substation secondary schemes. This can now be done without the need for a circuit outage (or if one is needed, its duration can be reduced) and the safety of personnel involved in commissioning, maintenance or retrofitting is also enhanced; i.e. secondary equipment renewals can be undertaken in a safer, quicker and easier way. It provides benefits for end users in terms of reduced costs and project duration and simplification of configuration changes required during the lifetime of a substation.

Protection and control flexibility is improved by using the process bus, since any changes only require modifications in the subscription of the protection IEDs, that receive the sampled values (SVs), rather than requiring hardwiring changes, as in the past. This will enable vendor and generation interoperability and simplify future changes in the secondary scheme, particularly when this involves software/firmware related reconfiguration or feature enhancement

The communication bus can be arranged in several different ways, depending on data flow requirements, reliability requirements or practicalities during installation. Figure 2 shows four alternative solutions of the process bus architectures.

Alternative 1 indicates a communication bus structure where each bay (installation unit) has its own process bus segment. To allow for protection and control equipment that requires data from more than one segment, a separate station-wide communication bus is installed, with switches or routers to each bay segment to transmit the required data streams.

Alternative 2 indicates a similar structure but each bay segment covers more than one bay. Data streams required by more than one segment are transferred by switches or routers. The example shows data from the busbar voltage transformer being used by directional earth-fault relays on all bays.

Alternative 3 indicates a single station-wide communications bus, to which all devices are connected. This requires a very high data rate on the bus, but eliminates the need for routers.

Alternative 4 indicates a function oriented bus structure. In this case the bus segments are set up to correspond to protection zones. Although routers are required, the segments can be arranged to minimize the data to be transferred between segments

The process bus has to be considered as a substation entity with defined interfaces. The process bus may therefore have properties such as reliability and performance that are reflected on the interface characteristics. The properties will be included in the substation 
design/integration, for example in reliability and performance calculations, as any other entity. The process bus entity may, and will, comprise several parts that vary by vendor and implementation.

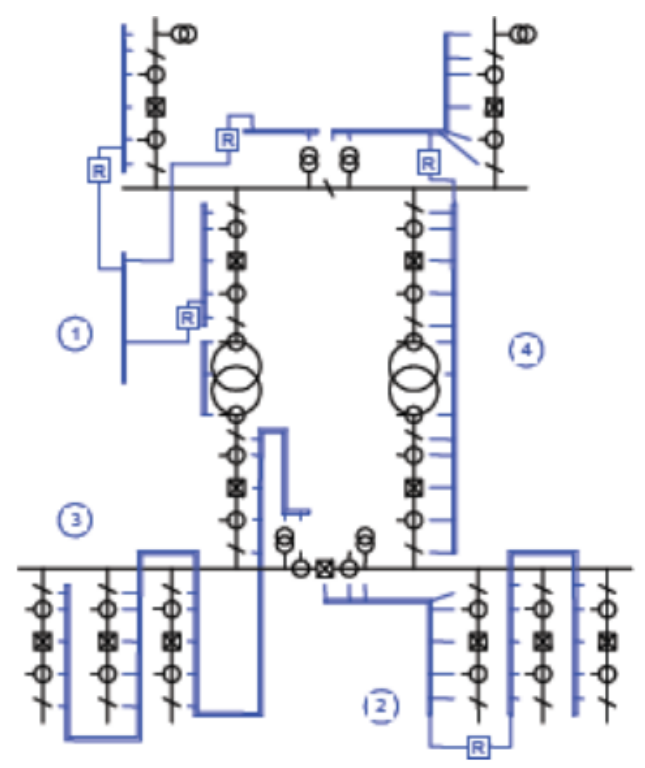

Figure 2 Alternative process bus architectures

\subsection{Process Bus Implementation}

Figure 3 illustrates substation automation with station and Process Bus. The red encircled portion of the Figure 3 is the main task of the research work where the Merging Unit is an interface between instrument transformers and the Process Bus. Subsequently, the Process Bus is the interface between the Merging Unit and IEDs.

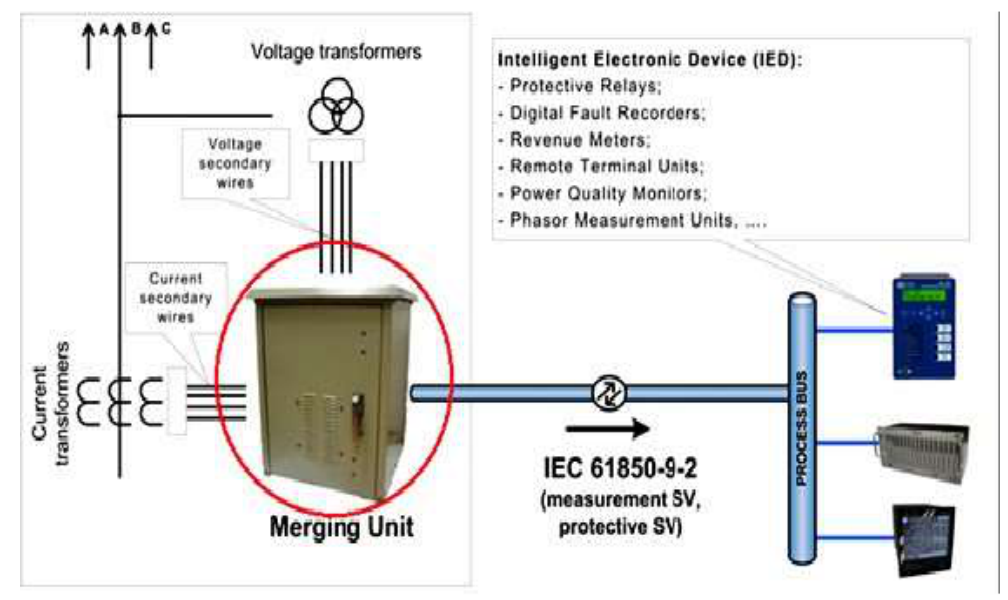

Figure 3 Substation Automation with Station and Process Bus.

The objective of this research is to have a logical Merging Unit which allows running the simulations and testing on a PC which eliminates the need for any vendor specific products, which are available at a high cost. The final goal of this research is the development of a Merging Unit that compiles with the IEC 61850 9-2LE standard, guidelines, understanding of its operation within CIT and the Process Bus. Figure 4 shows a complete Process Bus solution with logical Merging Unit. 


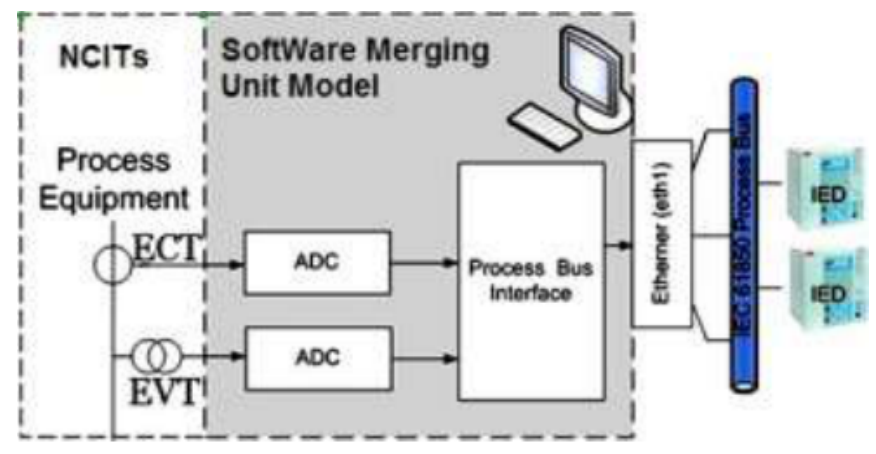

Figure 4 Complete Soft/Logical Merging Unit Model Project

\subsection{Methodologies and Techniques}

The research methodology and techniques are as follows:

- Describe the IEC 61850 standard and its significance especially IEC 61850 sub-clauses IEC 60044-8 and IEC 61850-9-2 about digital communication and Sampled Values transmission over Ethernet link

- Develop an understanding of IEC 61850 based SAS

- Study the UCA Implementation Guideline for IEC 61850-9- 2 (9-2 Light Edition or 9-2LE) which specifies Sampled Value data sets that are transmitted, sampling rates, time synchronisation requirements and physical interface.

- Research method for converting Analogue measurement signals from optical components to digital signals by the Merging Unit for digital interface between process level and bay level according to IEC 61850-9-2 LE guideline.

These digital signals will have the information of primary currents and voltages. The available literature in this area of research is dynamic and highly informative.

\subsection{IEC 61850}

Early times multiple protocols exist for substation automation, which include many protocols with custom communication links. Different vendor's devices Interoperability would be an advantage to/for user of substation automation device. About 60 members of IEC project group from different countries worked in IEC three working groups from 1995. After working on all the concerns and objectives, they created IEC 61850 standard. With the goal set for substation automation were:

- A single protocol for a complete substation allowing for the modelling of different data required for substation

- Classification of basic services required transferring data so that the complete mapping to communication protocol can be made future proof

- Support of high inter-operability between systems from different vendors

- A common method/format for storing complete data

- Define complete testing required for the equipment which conforms to the standard

To achieve Smart Grid vision outlined in the road map, the IEC 61850 is listed as a relevant and recommended standard. IEC 61850 with the help of modern Information and Communication Technology (ICT) to facilitate communication solution, Fibre optic or Registered Jack (RJ) cables, network switches and routers are used to reduce the copper wire. Digital information is transferred in a substation with the help of a microprocessor based device and automation solution which was absent in the past, which was the main reason 
behind holding back the digitisation of substation automation. Figure 5 shows digital substation automation system.

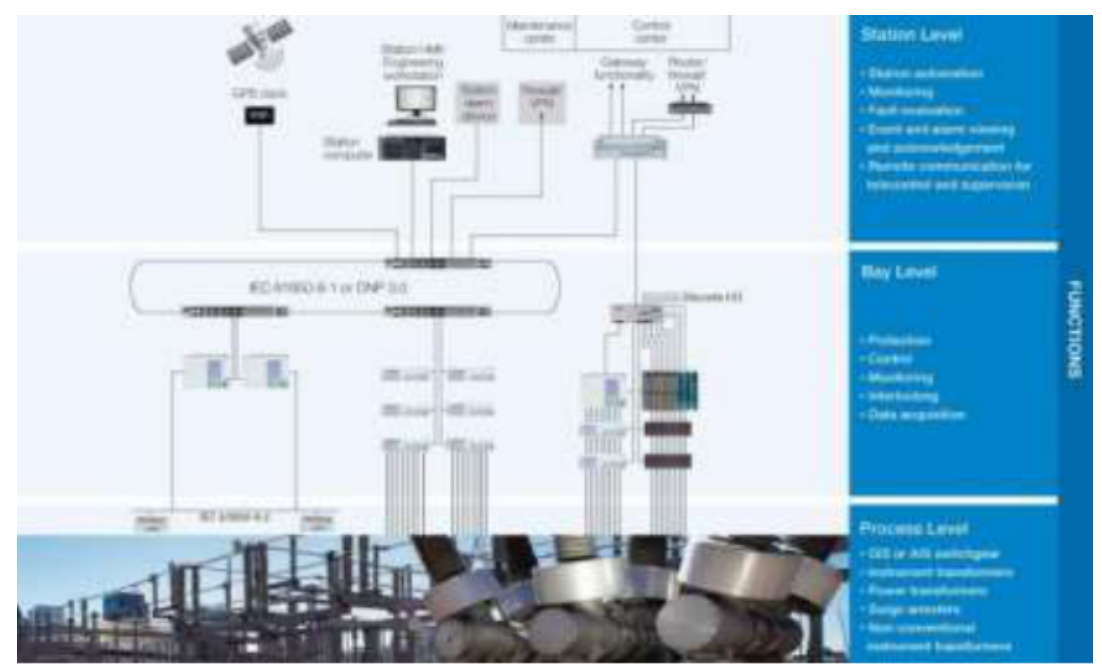

Figure 5 Digital Substation Automation System.

\section{THE IEC61850 BASED SUBSTATION}

\subsection{Functional hierarchy and interfaces for IEC61850}

The three levels of functional hierarchy of IEC 61850 are shown in Fig. 6. These include:-

The Process level: This level includes switchyard equipments such as CTs / VTs, Remote $\mathrm{I} / \mathrm{O}$, actuators, etc.

The Bay level: Bay level includes protection and control IEDs.

The Station level: Functions requiring information from all bays in the substation for control and reporting.

The process bus facilitates the time critical communication between protection and control IEDs to the primary equipment in the substation. This data includes sampled values, binary status signals or binary control signals.

The station bus provides communications between the station control, command and reporting level and the bay level. It also facilitates communications between different bays.

\subsection{Protection Philosophies}

Both the protection function within a power system substation and its associated communications bus structure must comply with the operating philosophies of the power company. Although there are standard rules across the industry, each company has developed its own set of rules dictated by its operations and its experience.

Grid transmission protection systems generally use a Main A Main B scheme in which should there be a failure in either system, the parallel system will ensure that the primary system has the required standard of protection. The Main A and Main B protection systems are required to be independent of each other. Any failure in either system should result in that system being taken out of service thus reducing the possibility of nuisance tripping.

7 Golden rules of Process Bus are:-

- All trip signals shall be received by the breakers within $10 \mathrm{~ms}$ (excluding inter-trip send).

- No single activity on the MAIN 1 system shall affect the MAIN 2 system. 
- No single failure shall result in the loss of control of more than one bay.

- Physical facilities shall be available to isolate a bay for testing (Protection \& Control).

- The Protection and Control application - philosophy shall be functionally identical to the current solution.

- The design principles must be standard for all bay types.

- The switching box should be located as close as possible to the Primary equipment

\subsection{Process Bus Design}

There are numerous bus designs that could be used for Ethernet local area networks. Different applications have subtly different requirements and customised solutions result.

The three basic configurations include the cascaded bus, the star bus and the ring bus.

The typical cascaded architecture is arguably the simplest in which each user is connected to the bus via a switch. The bus is configured by connecting each switch to the next switch in the sequence as required. The maximum number of switches which can be cascaded in the chain, depends on the worst case time delay that can be tolerated by the system.

In the star architecture, each component is directly connected to a common central node, a multiport Ethernet switch. The message transmission times are reduced since all communications is routed through this central point. However, since all IEDs are connected to a single central Ethernet switch which produces an inherent weakness in the scheme.

The ring architecture is very similar to cascaded architecture except that the chain of switches is closed to form the ring by connecting the last switch to the last switch. Being a ring structure gives it an inherent self-healing facility.

Standard Ethernet switches do not support "loops", since this could lead to messages circulating indefinitely round the ring and therefore use all the available bandwidth. Managed switches are required for this architecture. These take into consideration the potential for loops and use a Rapid Spanning Tree Protocol, RSTP, defined in the IEEE 802.1w. This protocol allows switches to detect loops and internally block messages from circulating around the loop. The advantage of these managed switches is that they enable the process bus to be reconfigured following a communication network fault.

Studies into the reliability of these communications architectures [8] have shown that the star architecture has the highest reliability due to the lower component count. The cascade and ring architectures have lower but similar first failure reliabilities. The self-healing ability of the ring architecture offers the ability to withstand a single failure.

This self-healing ability of the ring architecture can be argued to be contrary to the philosophy of the Main A - Main B protection concept. It is suggested that should any part of either the Main A or Main B protection scheme fail, that system should be taken out of service leaving the other to provide the required level of protection. A 'Process Bus failure' alarm would alert operators of the need for repair.

The above considerations led to the use of the star architecture with separate stars being used for Main A and Main B. The typical star configuration is shown in Fig. 6. 


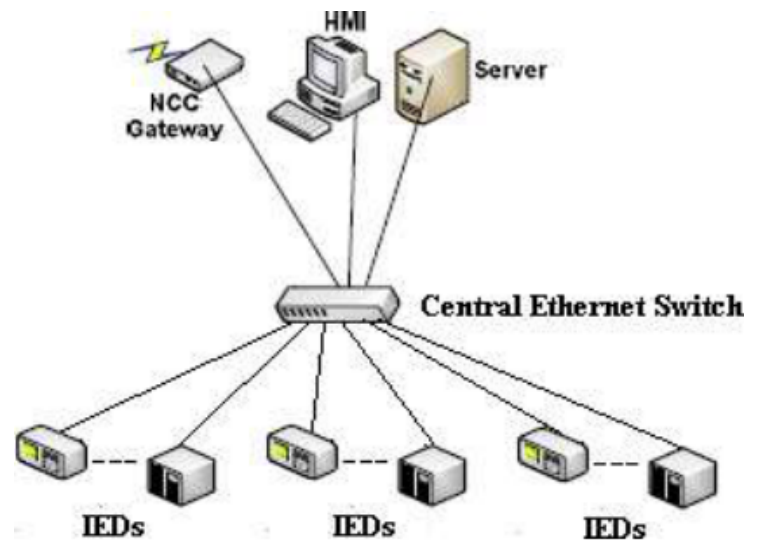

Figure 6 Star architecture process buses for Main A and Main B protection.

\section{CONCLUSIONS}

The introduction of the communications standard for the digital substation, IEC 61850, has provided the basis for the future protection and control systems. Based on the alternative communications bus architectures and the Company's operating performance requirements, the star structure was chosen to be examined.

The performance of an IEC61850 protection scheme using the star architecture was investigated with an RTDS real time simulator modelling an EHV transmission system. The particular scheme used a permissive under-reach protection scheme with an IEC61850 relay at the local end and a conventional relay, using the similar operating principles, at the remote end.

\section{REFERENCES}

[1] Yong-Cheol Kang, Seung-HunOk, Sang-Hee Kang, "A CT saturation detection algorithm”, IEEE Transaction on Power Delivery, Vol 19, pp 78-85, Jan 2018.

[2] Luoyun Xu, Haiyu Li , Linwei Chen, Christopher Patterson, Priyanka Mohapatra, "Assessment and analysis of different process bus redundancy networks performance for IEC61850- based digital substation”, IET Journels, eISSN, PP 2051-3305, 2018.

[3] K. Narendra, A. Dasgupta, K. Ponram, N. Perera, R. Midence, A. Oliveira, "Microprocessor based advanced bus protection scheme using iec 61850 process bus (9-2) sampled values", 2017 70 Annual Conference for Protective Relay Engineers (CPRE), Date of Conference: 3-6 April 2017.

[4] D. A. Barron, P Holliday, "Implementation of IEC61850 process bus. A utility view on design, installation, testing and commissioning, and lifetime issues". The 10th International Conference on Developments in Power System Protection, (2010).

[5] X Sun, M. A. Redfern, "Process bus configuration for protection schemes in the digital substation: IEC61850", The 46th International Universities' Power Engineering Conference, (2011). 